\title{
Hypoadiponectinemia is Associated With Impaired Glucose Tolerance and Coronary Artery Disease in Non-Diabetic Men
}

\author{
Fumiyuki Otsuka, MD; Seigo Sugiyama, MD; Sunao Kojima, MD; Hidetomo Maruyoshi, MD; \\ Tohru Funahashi, MD*; Tomohiro Sakamoto, MD; Michihiro Yoshimura, MD; \\ Kazuo Kimura, MD**; Satoshi Umemura, MD**; Hisao Ogawa, MD
}

\begin{abstract}
Background Impaired glucose tolerance (IGT) is a significant risk factor for cardiovascular disease, but is not always recognized in the clinical setting. An anti-atherogenic adipocytokine, adiponectin, is decreased in type 2 diabetes mellitus, but its role in non-diabetic subjects has not been clarified. The hypothesis investigated in the present study was that plasma adiponectin levels correlate with IGT and coronary artery disease (CAD) in nondiabetic men.

Methods and Results Glucose intolerance was evaluated by an oral glucose tolerance test and plasma adiponectin levels were measured in 232 non-diabetic men who underwent coronary angiography. Patients with IGT $(n=102)$ had significantly lower adiponectin levels than those with normal glucose tolerance $(n=130)(4.47$ [3.23-6.39] vs 5.85 [3.99-8.65] $\mathrm{g} / \mathrm{ml}, \mathrm{p}=0.003)$. Plasma adiponectin levels were associated with IGT in multiple logistic regression analysis (odds ratio (OR) 0.623 , 95\% confidence interval (CI) $0.397-0.980 ; \mathrm{p}=0.041$ ). Non-diabetic patients with CAD $(n=122)$ had lower adiponectin levels than those without CAD $(n=110)(4.60$ [3.32-6.38] vs 6.08 [4.10-9.88] $\mathrm{g} / \mathrm{ml}, \mathrm{p}<0.001)$. Multiple logistic regression analysis demonstrated adiponectin independently correlated with the presence of CAD (OR $0.432,95 \%$ CI $0.256-0.728 ; \mathrm{p}=0.002$ ).

Conclusions Hypoadiponectinemia is associated with IGT and CAD in non-diabetic men, suggesting that the adiponectin level can provide valuable information regarding the risk of CAD even in non-diabetic subjects. (Circ J 2007; 71: 1703-1709)
\end{abstract}

Key Words: Adiponectin; Coronary artery disease; Impaired glucose tolerance; Non-diabetic patients

$\mathbf{T}$ ype 2 diabetes mellitus (T2DM) is an established risk factor for cardiovascular disease, and is associated with a significantly worse prognosis in patients with coronary artery disease (CAD)! However, even in non-diabetic subjects, those with postprandial hyperglycemia are known to have an increased risk of cardiovascular events? Postprandial hyperglycemia in non-diabetic subjects is defined as impaired glucose tolerance (IGT) following an oral glucose tolerance test (OGTT), and IGT is considered a prestage of the onset of T2DM. Studies have revealed that IGT, but not impaired fasting glucose, is strongly associated with cardiovascular disease risk ${ }^{2,3}$ but IGT is not always recognized in the clinical setting because these patients often have normal fasting blood glucose levels and need an OGTT to be detected.

Adiponectin, an adipocyte-derived plasma protein, has antidiabetic properties and plays an important role in the development of T2DM. Plasma levels of adiponectin are

(Received May 6, 2007; revised manuscript received July 17, 2007; accepted July 30, 2007)

Department of Cardiovascular Medicine, Graduate School of Medical Sciences, Kumamoto University, Kumamoto, *Department of Internal Medicine and Molecular Science, Graduate School of Medicine, Osaka University, Suita and **Division of Cardiology, Yokohama City University Medical Center, Yokohama, Japan

Mailing address: Seigo Sugiyama, MD, Department of Cardiovascular Medicine, Graduate School of Medical Sciences, Kumamoto University, 1-1-1 Honjo, Kumamoto 860-8556, Japan. E-mail: ssugiyam @ kumamoto-u.ac.jp significantly decreased in patients with $\mathrm{T}_{2} \mathrm{DM}^{4}$ and conversely, high levels of adiponectin are independently associated with a reduced risk of the future onset of T2DM in apparently healthy subjects? In addition, there is increasing evidence that adiponectin has anti-atherogenic properties, and we recently reported a significant inverse association between plasma adiponectin levels and atherosclerotic burden? Low levels of adiponectin are known to be associated with $\mathrm{CAD}^{8}$ and increased risk of future myocardial infarction? Thus, hypoadiponectinemia is thought to be involved in the development of T2DM and atherosclerotic disease. However, the importance of adiponectin in nondiabetic subjects remains to be elucidated.

The hypothesis tested in the present study was that plasma levels of adiponectin are already decreased in patients with IGT and adiponectin levels can predict CAD in non-diabetic subjects. To investigate this, we evaluated glucose intolerance by an OGTT and measured plasma levels of adiponectin in non-diabetic men with or without CAD.

\section{Methods}

\section{Study Population}

The study subjects were non-diabetic men who underwent coronary angiography at Kumamoto University Hospital from January 2000 through June 2005 because of an abnormality on ECG or angina-like symptoms. All patients underwent a 75-g OGTT. Selective coronary angiography 
Table 1 Baseline Clinical Characteristics of 232 Non-Diabetic Men

\begin{tabular}{|c|c|c|c|}
\hline & $\begin{array}{c}N G T \\
(n=130)\end{array}$ & $\begin{array}{c}I G T \\
(n=102)\end{array}$ & $p$ value \\
\hline Age (years) & $66.3 \pm 9.4$ & $64.3 \pm 9.8$ & 0.115 \\
\hline$B M I\left(\mathrm{~kg} / \mathrm{m}^{2}\right)$ & $23.0 \pm 2.9$ & $24.4 \pm 2.9$ & $<0.001$ \\
\hline Current smoking & $37(28)$ & $24(24)$ & 0.454 \\
\hline Hypertension & $67(52)$ & $73(72)$ & 0.003 \\
\hline Systolic BP $(\mathrm{mmHg})$ & $128.3 \pm 18.6$ & $133.5 \pm 19.5$ & 0.039 \\
\hline Diastolic BP (mmHg) & $75.2 \pm 10.0$ & $78.9 \pm 12.4$ & 0.013 \\
\hline Total cholesterol $(\mathrm{mg} / \mathrm{dl})$ & $185.3 \pm 37.3$ & $188.9 \pm 33.3$ & 0.448 \\
\hline HDL-cholesterol $(\mathrm{mg} / \mathrm{dl})$ & $51.9 \pm 16.0$ & $47.5 \pm 12.8$ & 0.023 \\
\hline LDL-cholesterol $(\mathrm{mg} / \mathrm{dl})$ & $117.6 \pm 31.0$ & $122.9 \pm 30.9$ & 0.196 \\
\hline Triglycerides $(\mathrm{mg} / \mathrm{dl})$ & $99.0(70.0-131.5)$ & $123.0(88.0-160.0)$ & 0.003 \\
\hline Hypercholesterolemia & $55(43)$ & $49(48)$ & 0.492 \\
\hline Fasting glucose $(\mathrm{mg} / \mathrm{dl})$ & $88.1 \pm 7.9$ & $91.2 \pm 8.7$ & 0.005 \\
\hline Fasting insulin $(\mu U / m l)$ & $6.2 \pm 3.5$ & $7.8 \pm 4.8$ & 0.002 \\
\hline 2- $h$ glucose $(\mathrm{mg} / \mathrm{dl})$ & $104.9 \pm 23.0$ & $165.4 \pm 19.3$ & $<0.001$ \\
\hline 2-h insulin $\mu U / m l)$ & $48.0 \pm 40.2$ & $110.2 \pm 90.4$ & $<0.001$ \\
\hline Hemoglobin Alc (\%) & $5.27 \pm 0.40$ & $5.43 \pm 0.44$ & 0.005 \\
\hline HOMA-IR & $1.35 \pm 0.81$ & $1.77 \pm 1.11$ & $<0.001$ \\
\hline HOMA-ß (\%) & $97.3 \pm 67.4$ & $108.5 \pm 75.6$ & 0.234 \\
\hline Adiponectin $(\mu \mathrm{g} / \mathrm{ml})$ & $5.83(4.10-8.41)$ & $4.42(3.26-5.98)$ & $<0.001$ \\
\hline Creatinine ( $\mathrm{mg} / \mathrm{dl})$ & $0.94 \pm 0.22$ & $0.93 \pm 0.21$ & 0.656 \\
\hline$h s-C R P(m g / L)$ & $0.70(0.40-1.50)$ & $0.80(0.50-2.10)$ & 0.115 \\
\hline Family history of MI & $34(26)$ & $24(24)$ & 0.760 \\
\hline History of OMI & $16(12)$ & $20(20)$ & 0.146 \\
\hline $\operatorname{LVEF}(\%)$ & $64.0 \pm 14.9$ & $66.2 \pm 12.5$ & 0.253 \\
\hline$C A D$ & $59(45)$ & $63(62)$ & 0.019 \\
\hline \multicolumn{4}{|l|}{ Medications } \\
\hline$\beta$-blockers & $25(19)$ & $13(13)$ & 0.252 \\
\hline Calcium antagonists & $62(48)$ & $65(64)$ & 0.021 \\
\hline ACE inhibitors & $31(24)$ & $27(26)$ & 0.760 \\
\hline$A R B$ & $17(13)$ & $17(17)$ & 0.562 \\
\hline Nitrates & $29(22)$ & $31(30)$ & 0.213 \\
\hline Aspirin & $59(45)$ & $57(56)$ & 0.146 \\
\hline HMG-CoA reductase inhibitors & $27(21)$ & $28(27)$ & 0.302 \\
\hline
\end{tabular}

Values are mean $\pm S D$ or median value ( $25^{\text {th }}$ to $75^{\text {th }}$ percentile range) or $n(\%)$.

NGT, normal glucose tolerance; IGT, impaired glucose tolerance; BMI, body mass index; BP, blood pressure; HDL, high-density lipoprotein; LDL, low-density lipoprotein; HOMA-IR, homeostasis model assessment of insulin resistance; HOMA- $\beta$, homeostasis model assessment of pancreatic $\beta$-cell function; hs-CRP, high-sensitivity $C$-reactive protein; MI, myocardial infarction; OMI, old MI; LVEF, left ventricular ejection fraction; $C A D$, coronary artery disease; $A C E$, angiotensin-converting enzyme; ARBs, angiotensin II receptor blockers.

a

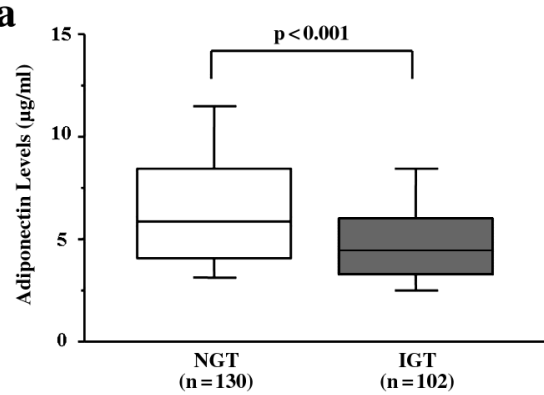

c

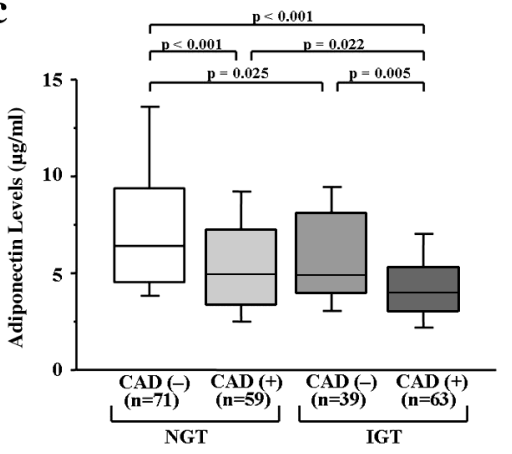

b

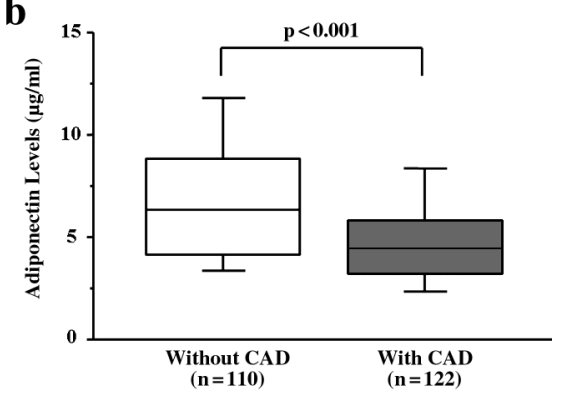

Fig 1. (a) Box-and-whisker plot showing plasma levels of adiponectin in men with normal glucose tolerance (NGT) and impaired glucose tolerance (IGT). (b) Boxand-whisker plot showing plasma levels of adiponectin in non-diabetic men with $(\mathrm{n}=$ $122)$ and without $(n=110)$ coronary artery disease (CAD). (c) Box-and-whisker plot showing plasma levels of adiponectin in men with NGT and IGT, with and without CAD. In these plots, lines within boxes represent median values; the upper and lower borders of the boxes represent the $75^{\text {th }}$ and $25^{\text {th }}$ percentiles, respectively; and the upper and lower bars outside the boxes represent the $90^{\text {th }}$ and $10^{\text {th }}$ percentiles, respectively. 
Table 2 Logistic Regression Analysis for IGT in Non-Diabetic Men

\begin{tabular}{lccccc}
\hline \hline \multirow{2}{*}{ Factor } & \multicolumn{2}{c}{ Simple regression } & & \multicolumn{2}{c}{ Multiple regression } \\
\cline { 2 - 3 } \cline { 5 - 6 } & OR $(95 \%$ CI $)$ & $p$ value & & OR $(95 \%$ CI $)$ & p value \\
\hline Age (per year) & $0.978(0.952-1.005)$ & 0.115 & & $0.989(0.958-1.020)$ & 0.485 \\
BMI (per kg/m ${ }^{2}$ ) & $1.187(1.078-1.306)$ & 0.001 & & $1.092(0.978-1.220)$ & 0.116 \\
Current smoking (yes) & $0.773(0.426-1.403)$ & 0.398 & & & \\
Hypertension (yes) & $2.367(1.364-4.106)$ & 0.002 & & $2.183(1.204-3.957)$ & 0.010 \\
Total cholesterol (per mg/dl) & $1.003(0.996-1.010)$ & 0.446 & & & \\
HDL-cholesterol (per mg/dl) & $0.978(0.959-0.997)$ & 0.026 & & $0.993(0.972-1.015)$ & 0.521 \\
LDL-cholesterol (per mg/dl) & $1.006(0.997-1.014)$ & 0.196 & & & \\
Triglycerides (per doubling) & $1.760(1.165-2.660)$ & 0.007 & & $1.275(0.649-2.503)$ & 0.481 \\
Fasting glucose (per mg/dl) & $1.047(1.014-1.082)$ & 0.005 & & $1.034(0.998-1.071)$ & 0.066 \\
Adiponectin (per doubling) & $0.464(0.315-0.684)$ & $<0.001$ & & $0.623(0.397-0.980)$ & 0.041 \\
hs-CRP (per doubling) & $1.125(0.954-1.327)$ & 0.160 & & & \\
\hline
\end{tabular}

OR, odds ratio; CI, confidence interval. Other abbreviations as in Table 1.

was performed using the Judkins technique, and CAD was defined as an organic stenosis $\geq 75 \%$ in at least 1 of the major coronary arteries. Patients with acute coronary syndromes were excluded from the study, as were patients with malignant disease, infectious disease, chronic inflammatory disease, autoimmune disease, and advanced renal disease (serum creatinine level $>2.5 \mathrm{mg} / \mathrm{dl}$ ). Among 269 men fulfilling the criteria, 37 were excluded because they were diagnosed as having DM following an OGTT. Accordingly, this study enrolled 232 consecutive non-diabetic men. Written informed consent was given by each patient before participation in the study. The study was performed in accordance with the ethics principles in the Declaration of Helsinki and the study protocol was approved by the ethics committee of our institution.

\section{OGTT}

A 75-g OGTT was performed after an overnight fast in order to identify patients with IGT or normal glucose tolerance (NGT) as defined by the criteria of the World Health Organization (IGT: fasting glucose level $<126 \mathrm{mg} / \mathrm{dl}$, $140 \mathrm{mg} / \mathrm{dl} \leq 2-\mathrm{h}$ post-load glucose level $<200 \mathrm{mg} / \mathrm{dl}$; NGT: fasting glucose level $<110 \mathrm{mg} / \mathrm{dl}$, 2-h post-load glucose level $<140 \mathrm{mg} / \mathrm{dl}) !^{10}$ The homeostasis model assessment of insulin resistance (HOMA-IR) was calculated as: HOMAIR $(\mu \mathrm{U} / \mathrm{ml} \times \mathrm{mg} / \mathrm{dl})=($ fasting insulin $\times$ fasting glucose $) /$ $405 !^{11}$ The homeostasis model assessment of pancreatic $\beta$ cell function (HOMA- $\beta$ ) was calculated as: HOMA- $\beta$ $[\mu \mathrm{U} / \mathrm{ml} /(\mathrm{mg} / \mathrm{dl})]=($ fasting insulin $\times 360) /($ fasting glucose 63)!1

\section{Blood Sampling and Measurement of Plasma Adiponectin}

Venous blood samples were obtained in the early morning from all subjects after a 12-h fast. Plasma samples were immediately stored at $-80^{\circ} \mathrm{C}$ for subsequent assay of adiponectin levels by enzyme-linked immunosorbent assay (adiponectin ELISA kit, Otsuka Pharmaceutical, Tokyo, Japan) as described previously?,12

\section{Statistical Analysis}

Results for normally distributed continuous variables are expressed as the mean value $\pm \mathrm{SD}$, and those for continuous variables with skewed distributions are expressed as the median value (interquartile range). Comparisons of continuous variables were analyzed with the unpaired t-test and the Mann-Whitney U-test, as appropriate. Categorical variables are presented by frequency counts, and intergroup comparisons were analyzed by $\mathrm{ax}^{2}$ test. Associations between the presence of IGT and CAD risk factors including highsensitivity C-reactive protein (hs-CRP), and those between the presence of CAD and all other parameters were first analyzed by simple logistic regression analysis and then by multivariate analysis, in which age and significant factors in the univariate analysis were entered. The base- 2 logarithms $\left(\log _{2}\right)$ of the plasma levels of adiponectin, triglycerides, and hs-CRP were used in all the logistic regression analyses to account for a skewed distribution of these parameters 13 Thus, odds ratios (ORs) for these variables reflect the change in odds for an increase of $1 \log _{2}$ (the equivalent of a doubling of the value) in the measured value. Comparisons of plasma levels of adiponectin in patients with NGT or IGT, with or without CAD, were assessed by the Kruskal-Wallis and the Mann-Whitney U-tests. Incremental effects on the prevalence of CAD of the combination of hypercholesterolemia and lower levels of adiponectin (or IGT) were also evaluated by $\mathrm{ax}^{2}$ test. Hypercholesterolemia was defined as low-density lipoprotein-cholesterol levels $>140 \mathrm{mg} / \mathrm{dl}$ or use of HMG-CoA reductase inhibitors, and lower levels of adiponectin were arbitrarily defined as $<5.00 \mu \mathrm{g} / \mathrm{ml}$, the $50^{\text {th }}$ percentile of the distribution of adiponectin levels in the study patients. Statistical significance was defined as $\mathrm{p}<0.05$. All analyses were performed using Stat View-5.0 software (SAS Institute Inc, Cary, NC, USA).

\section{Results}

\section{Adiponectin and IGT}

The baseline clinical characteristics of the 232 nondiabetic men are summarized in Table 1. Patients with IGT $(n=102)$ had a significantly higher body mass index (BMI), incidence of hypertension, triglycerides, fasting glucose, fasting insulin, hemoglobin A1c (HbA1c), HOMA-IR, and prevalence of CAD, and a significantly lower high-density lipoprotein (HDL)-cholesterol level than patients with NGT (Table 1). Plasma levels of adiponectin in patients with IGT were significantly lower than those in patients with NGT $(4.42[3.26-5.98]$ vs $5.83[4.10-8.41] \mu \mathrm{g} / \mathrm{ml}, \mathrm{p}<0.001)$ (Table 1; Fig 1a). In simple logistic regression analysis, BMI, hypertension, HDL-cholesterol, triglycerides, fasting glucose, and plasma adiponectin levels were significantly associated with IGT (Table 2). Multiple logistic regression analysis demonstrated that the plasma level of adiponectin was a significant independent determinant of IGT (OR $0.623,95 \%$ confidence interval $(\mathrm{CI}) 0.397-0.980 ; \mathrm{p}=0.041$ ) (Table 2). In addition to the fasting glucose level, other glucose tolerance-related variables such as fasting insulin 
Table 3 Logistic Regression Analysis for CAD in Non-Diabetic Men

\begin{tabular}{|c|c|c|c|c|}
\hline \multirow{2}{*}{ Factor } & \multicolumn{2}{|c|}{ Simple regression } & \multicolumn{2}{|c|}{ Multiple regression } \\
\hline & OR $(95 \% C I)$ & $p$ value & OR $(95 \% C I)$ & $p$ value \\
\hline Age (per year) & $1.047(1.018-1.077)$ & 0.001 & $1.091(1.050-1.131)$ & $<0.001$ \\
\hline$B M I\left(\right.$ per $\left.\mathrm{kg} / \mathrm{m}^{2}\right)$ & $1.077(0.986-1.177)$ & 0.098 & & \\
\hline Current smoking (yes) & $0.760(0.423-1.365)$ & 0.359 & & \\
\hline Hypertension (yes) & $2.477(1.444-4.249)$ & 0.001 & $2.066(1.043-4.090)$ & 0.037 \\
\hline Total cholesterol (per mg/dl) & $1.001(0.994-1.008)$ & 0.835 & & \\
\hline HDL-cholesterol (per mg/dl) & $0.963(0.942-0.983)$ & $<0.001$ & $0.973(0.949-0.998)$ & 0.033 \\
\hline LDL-cholesterol (per mg/dl) & $1.004(0.996-1.013)$ & 0.319 & & \\
\hline Triglycerides (per doubling) & $1.757(1.161-2.660)$ & 0.008 & $1.496(0.869-2.576)$ & 0.146 \\
\hline Hypercholesterolemia (yes) & $3.749(2.159-6.507)$ & $<0.001$ & $3.202(1.657-6.187)$ & $<0.001$ \\
\hline Fasting glucose (per mg/dl) & $1.015(0.984-1.047)$ & 0.344 & & \\
\hline Fasting insulin (per $\mu U / m l)$ & $1.043(0.978-1.112)$ & 0.202 & & \\
\hline 2- $h$ glucose $($ per $m g / d l)$ & $1.009(1.001-1.016)$ & 0.020 & $1.001(0.991-1.011)$ & 0.805 \\
\hline 2-h insulin $($ per $\mu U / m l)$ & $1.004(1.000-1.007)$ & 0.073 & & \\
\hline Hemoglobin Alc (per \%) & $1.166(0.624-2.179)$ & 0.631 & & \\
\hline HOMA-IR (per count) & $1.231(0.929-1.632)$ & 0.147 & & \\
\hline HOMA- $\beta$ (per \%) & $0.999(0.996-1.003)$ & 0.755 & & \\
\hline Adionectin (per doubling) & $0.366(0.244-0.550)$ & $<0.001$ & $0.432(0.256-0.728)$ & 0.002 \\
\hline hs-CRP (per doubling) & $1.189(1.005-1.406)$ & 0.043 & $1.145(0.927-1.414)$ & 0.208 \\
\hline Family history of MI (yes) & $1.714(0.932-3.152)$ & 0.083 & & \\
\hline
\end{tabular}

Abbreviations as in Tables 1,2.

a

b

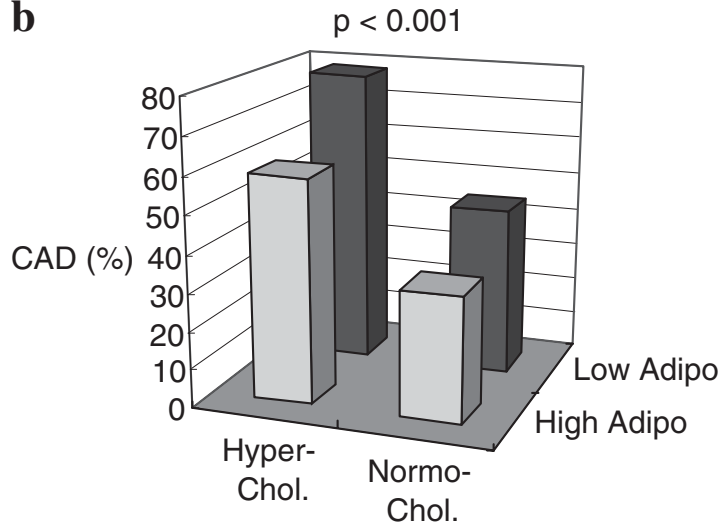

Fig 2. (a) Bar graph showing incremental effect on the prevalence of coronary artery disease (CAD) of the combination of hypercholesterolemia (low-density lipoprotein-cholesterol levels $>140 \mathrm{mg} / \mathrm{dl}$ or use of HMG-CoA reductase inhibitors) and impaired glucose tolerance (IGT). (b) Bar graph showing incremental effect on the prevalence of CAD of the combination of hypercholesterolemia and lower levels of adiponectin $\left(<5.00 \mu \mathrm{g} / \mathrm{ml}\right.$; arbitrarily defined as the $50^{\text {th }}$ percentile of the distribution of adiponectin levels in the study patients). NGT, normal glucose tolerance; Hyper-Chol, hypercholesterolemia; NormoChol, normocholesterolemia; Low Adipo, low levels of adiponectin; High Adipo, high levels of adiponectin.
(OR 1.110, 95\% CI 1.034-1.191; $\mathrm{p}=0.004)$, and HbAlc (OR $2.550,95 \%$ CI $1.315-4.944 ; \mathrm{p}=0.006)$ were also significantly associated with IGT in simple logistic regression analysis. Multivariate analysis including these variables and the adiponectin levels revealed that $\mathrm{HbA} 1 \mathrm{c}$ (OR 2.258, 95\% CI 1.088-4.688; $\mathrm{p}=0.029)$ and adiponectin (OR 0.532, 95\% CI $0.352-0.805 ; \mathrm{p}=0.003$ ) were significantly and independently associated with IGT.

\section{Adiponectin and CAD in Non-Diabetic Men}

Non-diabetic patients with CAD $(n=122)$ had significantly lower adiponectin levels than those without CAD $(n=$ 110) $(4.45$ [3.17-5.81] vs 6.32 [4.17-8.79] $\mathrm{\mu} / \mathrm{ml}, \mathrm{p}<0.001)$ (Fig 1b). Simple logistic regression analysis showed that age, hypertension, HDL-cholesterol, triglycerides, hypercholesterolemia, 2-h glucose, hs-CRP, and plasma adiponectin level were associated with $\mathrm{CAD}$ in non-diabetic men (Table 3). Multiple logistic regression analysis revealed that age (OR 1.091, 95\% CI 1.050-1.131; $\mathrm{p}<0.001)$, hypertension (OR 2.066, 95\% CI 1.043-4.090; $\mathrm{p}=0.037$ ), HDLcholesterol (OR 0.973, 95\% CI 0.949-0.998; p=0.033), hypercholesterolemia (OR 3.202, 95\% CI 1.657-6.187; $\mathrm{p}<$ 0.001 ), and plasma adiponectin levels (OR $0.432,95 \%$ CI $0.256-0.728 ; \mathrm{p}=0.002$ ) significantly and independently correlated with the presence of CAD in non-diabetic men (Table 3).

\section{Adiponectin and CAD in Men With NGT or IGT}

Among 130 men with NGT, those with CAD $(n=59)$ had significantly lower plasma levels of adiponectin than those without CAD $(n=71)(4.91$ [3.40-7.24] vs 6.42 [4.55-9.35] $\mu \mathrm{g} / \mathrm{ml}, \mathrm{p}<0.001$ ) (Fig 1c). Moreover, among 102 men with IGT, plasma levels of adiponectin in the men with CAD $(n=63)$ were also significantly lower than in the men without CAD $(n=39)(3.97$ [3.02-5.28] vs 4.91 [4.00-8.14] $\mu \mathrm{g} / \mathrm{ml}, \mathrm{p}=0.005$ ) (Fig 1c). Adiponectin levels in NGT patients with CAD were comparable to those in IGT patients without CAD.

Simple logistic regression analysis among the patients with NGT and those with IGT showed that plasma adiponectin levels were significantly associated with CAD (NGT: 
crude OR $0.413,95 \%$ CI $0.245-0.695, \mathrm{p}=0.001$; IGT: crude OR $0.349,95 \%$ CI $0.175-0.694, \mathrm{p}=0.003$ ). After adjustment for age, BMI, smoking status, hypertension, HDL-cholesterol, triglyceride levels, and hypercholesterolemia, plasma levels of adiponectin remained a significant determinant of the presence of CAD in both groups of patients (NGT: adjusted OR $0.477,95 \%$ CI $0.248-0.916, p=0.026$; IGT: adjusted OR $0.237,95 \%$ CI $0.090-0.621, \mathrm{p}=0.003$ ).

\section{IGT, Lower Adiponectin, and CAD}

Among 232 non-diabetic men, IGT was significantly associated with CAD in simple logistic regression analysis (OR 1.944, 95\% CI 1.147-3.296; p=0.014). After adjustment for hypercholesterolemia, IGT remained a significant determinant of the presence of CAD in non-diabetic men (OR 1.929, 95\% CI 1.106-3.364; $\mathrm{p}=0.021$ ). In addition, IGT in combination with hypercholesterolemia showed a significant incremental effect on the risk of CAD in nondiabetic men $(\mathrm{p}<0.001)$ (Fig 2a).

When the cut-off value of adiponectin levels was defined as $5.00 \mathrm{\mu} \mathrm{g} / \mathrm{ml}$ (the $50^{\text {th }}$ percentile of the distribution in the study patients), lower levels of adiponectin were significantly associated with CAD in simple logistic regression analysis (OR 2.160, 95\% CI 1.278-3.652; $\mathrm{p}=0.004)$. Moreover, lower levels of adiponectin in combination with hypercholesterolemia demonstrated a significant incremental effect on the risk of $\mathrm{CAD}(\mathrm{p}<0.001)$ (Fig $2 \mathrm{~b})$.

\section{Discussion}

In the present study, we demonstrated that men with IGT had significantly lower plasma adiponectin levels than those with NGT, and that non-diabetic men with CAD had significantly lower plasma adiponectin levels than those without CAD. Moreover, the plasma level of adiponectin was an independent determinant of IGT and CAD in nondiabetic men. These results suggest that plasma levels of adiponectin are already decreased in patients with IGT before the onset of overt diabetes, and that adiponectin levels may be a useful marker for identifying patients at high risk for $\mathrm{CAD}$ even in non-diabetic subjects.

It is well established that T2DM is a significant risk factor for cardiovascular disease; however, non-diabetic subjects with postprandial hyperglycemia (which is defined as IGT) are also at higher risk of cardiovascular disease?,3 Previous studies demonstrated that adiponectin levels are decreased in patients with $\mathrm{T}^{2} \mathrm{DM}^{4}$ and $\mathrm{CAD}$, including vasospastic angina, 84,15 Individuals with high concentrations of adiponectin are less likely to develop T2DM5, and lower adiponectin levels are significantly associated with the future development of myocardial infarction? Although these observations indicate that low levels of adiponectin may contribute to the development of T2DM and atherosclerotic disease, the importance of adiponectin in nondiabetic subjects has not been elucidated. In Pima Indians, it has been shown that adiponectin levels in subjects with IGT and early diabetes ( $\leq 10$ years duration) were lower than in subjects with NGT!6 A recent study in Japanese Americans showed lower levels of adiponectin in IGT subjects than in NGT subjects, although the difference was not statistically significant ${ }^{17}$ However, those studies included both men and women, and there is a significant gender difference in adiponectin levels ${ }^{12}$ Furthermore, precise evaluation including the presence or absence of CAD in non-diabetic subjects has not been performed.
In this study, we showed that men with IGT had significantly lower levels of adiponectin than those with NGT. Previous studies demonstrated a significant association between insulin resistance and IGT; 18 however, the precise pathophysiology of IGT has not been well clarified. It is well known that plasma levels of adiponectin decrease with the accumulation of visceral adipose tissue, ${ }^{6}$ and in rhesus monkeys, adiponectin is reduced in parallel with progression of insulin resistance.$^{9}$ In addition, increased serum concentrations of adiponectin have been shown to be associated with increased insulin sensitivity and glucose tolerance in humans $2^{20}$ Thus, decreased levels of adiponectin concomitant with visceral fat accumulation may lead to insulin resistance and the development of IGT, which is considered a pre-stage of T2DM.

We also demonstrated that non-diabetic men with CAD had significantly lower levels of adiponectin than those without $\mathrm{CAD}$, and that the adiponectin level was an independent determinant of CAD, even in non-diabetic men. Although the precise mechanism of decreased adiponectin levels in patients with CAD has not been elucidated, it is conceivable that reduced levels of this anti-atherogenic molecule may lead to the development of atherosclerosis. Adiponectin could reduce atherosclerosis by inhibiting endothelial expression of adhesion molecules, ${ }^{21}$ macrophage-to-foam cell transformation ${ }^{2} 2$ and vascular smooth muscle cell proliferation. ${ }^{23}$ In addition, we have reported a reduction of plasma adiponectin in the early phase of acute myocardial infarction ${ }^{24}$ and accumulation of adiponectin has been demonstrated in the walls of injured vessels but not in intact vessels 22 Therefore, increased consumption of circulating adiponectin in active atheroma might also bring about the reduction of adiponectin levels in patients with CAD.

Several lines of evidence demonstrate that IGT itself is a significant risk factor for cardiovascular disease. However, IGT is often not diagnosed in the clinical setting because of nearly normal fasting glucose levels and not performing an OGTT. In 2001, the National Cholesterol Education Program Adult Treatment Panel III introduced clinical criteria for defining the metabolic syndrome 25 in which fasting glucose levels were used to identify patients with glucose intolerance, because an OGTT is not routinely performed in the clinical setting. However, it has been shown that impaired fasting glucose is not significantly associated with cardiovascular events, ${ }^{2,3}$ Indeed, in the present study, which focused on non-diabetic subjects, the fasting blood glucose level was not a significant determinant of CAD in logistic regression analysis, whereas IGT was significantly associated with CAD. In identifying patients at high risk for CAD among non-diabetic subjects, it is important to detect IGT in addition to hypercholesterolemia, an established risk factor for CAD. In this study, we demonstrated a significant incremental effect on the prevalence of CAD of the combination of hypercholesterolemia and IGT, and similarly, hypercholesterolemia and hypoadiponectinemia. From the viewpoint of CAD risk stratification in non-diabetic subjects, the significant association between adiponectin and IGT demonstrated in this study suggests that the measurement of adiponectin levels is a practical screening tool for IGT in the clinical setting, and can provide valuable information regarding the risk of CAD in non-diabetic subjects.

It has been reported that previously undiagnosed DM and IGT are frequently observed in patients with acute myo- 
cardial infarction at hospital admission, ${ }^{26}$ and such newly detected abnormal glucose tolerance is a strong predictor for future cardiovascular events. ${ }^{27}$ Importantly, the treatment of IGT may prevent the development of overt diabetes 28 and IGT may be a modifiable risk factor for cardiovascular disease. A recent clinical study in Hispanic women with prior gestational diabetes demonstrated that treatment with thiazolidinedione, a peroxisome proliferator-activated receptor- $\gamma$ agonist, preserved pancreatic $\beta$-cell function and reduced the risk of the development of T2DM $2^{29}$ Our results suggest that there might be therapeutic potential of thiazolidinedione, which is known to increase adiponectin 30 in patients with IGT and hypoadiponectinemia. Increasing adiponectin levels by thiazolidinedione may be a potentially useful therapeutic strategy to improve glucose tolerance and reduce the risk of cardiovascular events, even in nondiabetic subjects with hypoadiponectinemia. However, further prospective clinical studies are required to clarify these issues because we did not evaluate the effect of such agents in the present study.

The major limitations of this study are the relatively small sample size, as well as the specific study subjects who underwent coronary angiography. The present study population might not represent ordinal non-diabetic subjects, and thus further studies in a large number of general subjects are required to confirm our results. The definition of CAD in this study was an angiographic documented organic stenosis of $\geq 75 \%$; however, it is currently recognized that evaluation of coronary plaque instability in addition to the degree of luminal stenosis, is important in assessing the risk of acute cardiovascular complications in patients with CAD. Thus, it is unclear whether the measurement of plasma adiponectin levels can be useful in predicting acute vascular complications in non-diabetic subjects. Moreover, we could not gather all the data regarding waist and hip circumferences, which reflect visceral and subcutaneous adiposity. Visceral fat accumulation is thought to cause insulin resistance, and whether lower levels of adiponectin correlate with IGT, independent of waist circumference, needs to be further evaluated.

In conclusion, men with IGT had significantly lower plasma adiponectin levels than those with NGT, and the adiponectin level was an independent determinant of CAD in non-diabetic men. Hypoadiponectinemia is significantly associated with IGT and CAD in non-diabetic men, and our results suggest that the measurement of adiponectin levels would be useful for the evaluation of IGT and provide valuable information regarding the risk of CAD even in non-diabetic subjects.

\section{Acknowledgments}

The authors thank Sachiyo Tanaka for the excellent technical support. This study was supported by a Research Grant for Cardiovascular Disease (17C-2) from the Ministry of Health, Labor and Welfare, Japan, a Grant-in-aid for Scientific Research (B-17390232, C-17590753, and C18590780) from the Ministry of Education, Culture, Sports, Science and Technology, Japan, and the Smoking Research Foundation Grant for Biomedical Research, Japan.

\section{References}

1. Norhammar A, Malmberg K, Diderholm E, Lagerqvist B, Lindahl B, Ryden L, et al. Diabetes mellitus: The major risk factor in unstable coronary artery disease even after consideration of the extent of coronary artery disease and benefits of revascularization. $J$ Am Coll Cardiol 2004; 43: 585-591.

2. Glucose tolerance and mortality: Comparison of WHO and American
Diabetes Association diagnostic criteria. The DECODE study group. European Diabetes Epidemiology Group. Diabetes epidemiology: Collaborative analysis of diagnostic criteria in Europe. Lancet 1999; 354: $617-621$.

3. Blake DR, Meigs JB, Muller DC, Najjar SS, Andres R, Nathan DM. Impaired glucose tolerance, but not impaired fasting glucose, is associated with increased levels of coronary heart disease risk factors: Results from the Baltimore Longitudinal Study on Aging. Diabetes 2004; 53: 2095-2100.

4. Hotta K, Funahashi T, Arita Y, Takahashi M, Matsuda M, Okamoto $\mathrm{Y}$, et al. Plasma concentrations of a novel, adipose-specific protein, adiponectin, in type 2 diabetic patients. Arterioscler Thromb Vasc Biol 2000; 20: $1595-1599$.

5. Spranger J, Kroke A, Mohlig M, Bergmann MM, Ristow M, Boeing $\mathrm{H}$, et al. Adiponectin and protection against type 2 diabetes mellitus. Lancet 2003; 361: 226-228.

6. Matsuzawa Y, Funahashi T, Kihara S, Shimomura I. Adiponectin and metabolic syndrome. Arterioscler Thromb Vasc Biol 2004; 24: $29-33$.

7. Kojima S, Funahashi T, Maruyoshi H, Honda O, Sugiyama S, Kawano H, et al. Levels of the adipocyte-derived plasma protein, adiponectin, have a close relationship with atheroma. Thromb Res 2005; 115: 483-490.

8. Kumada M, Kihara S, Sumitsuji S, Kawamoto T, Matsumoto S, Ouchi N, et al. Association of hypoadiponectinemia with coronary artery disease in men. Arterioscler Thromb Vasc Biol 2003; 23: 8589.

9. Pischon T, Girman CJ, Hotamisligil GS, Rifai N, Hu FB, Rimm EB. Plasma adiponectin levels and risk of myocardial infarction in men. JAMA 2004; 291: 1730-1737.

10. Alberti KG, Zimmet PZ. Definition, diagnosis and classification of diabetes mellitus and its complications. Part 1: Diagnosis and classification of diabetes mellitus provisional report of a $\mathrm{WHO}$ consultation. Diabet Med 1998; 15: 539-553.

11. Matthews DR, Hosker JP, Rudenski AS, Naylor BA, Treacher DF, Turner RC. Homeostasis model assessment: Insulin resistance and beta-cell function from fasting plasma glucose and insulin concentrations in man. Diabetologia 1985; 28: 412-419.

12. Arita Y, Kihara S, Ouchi N, Takahashi M, Maeda K, Miyagawa J, et al. Paradoxical decrease of an adipose-specific protein, adiponectin, in obesity. Biochem Biophys Res Commun 1999; 257: 79-83.

13. Tsimikas S, Brilakis ES, Miller ER, McConnell JP, Lennon RJ, Kornman KS, et al. Oxidized phospholipids, Lp(a) lipoprotein, and coronary artery disease. $N$ Engl J Med 2005; 353: 46-57.

14. Maruyoshi H, Kojima S, Otsuka F, Funahashi T, Kaikita K, Sugiyama $\mathrm{S}$, et al. Hypoadiponectinemia is associated with coronary artery spasm in men. Circ J 2005; 69: 1154-1156.

15. Hong SJ, Seo HS, Rha SW, Oh DJ, Kwon JA, Lee KN, et al. Decrease in plasma adiponectin concentrations in patients with variant angina pectoris. Circ J 2006; 70: 414-418.

16. Looker HC, Krakoff J, Funahashi T, Matsuzawa Y, Tanaka S, Nelson $\mathrm{RG}$, et al. Adiponectin concentrations are influenced by renal function and diabetes duration in Pima Indians with type 2 diabetes. J Clin Endocrinol Metab 2004; 89: 4010-4017.

17. Nakanishi S, Yamane K, Kamei N, Nojima H, Okubo M, Kohno N. A protective effect of adiponectin against oxidative stress in Japanese Americans: The association between adiponectin or leptin and urinary isoprostane. Metabolism 2005; 54: 194-199.

18. Sacca L, Orofino G, Petrone A, Vigorito C. Differential roles of splanchnic and peripheral tissues in the pathogenesis of impaired glucose tolerance. J Clin Invest 1984; 73: 1683-1687.

19. Hotta K, Funahashi T, Bodkin NL, Ortmeyer HK, Arita Y, Hansen $\mathrm{BC}$, et al. Circulating concentrations of the adipocyte protein adiponectin are decreased in parallel with reduced insulin sensitivity during the progression to type 2 diabetes in rhesus monkeys. Diabetes 2001; 50: $1126-1133$.

20. Goldfine AB, Kahn CR. Adiponectin: Linking the fat cell to insulin sensitivity. Lancet 2003; 362: 1431-1432.

21. Ouchi N, Kihara S, Arita Y, Maeda K, Kuriyama H, Okamoto Y, et al. Novel modulator for endothelial adhesion molecules: Adipocytederived plasma protein adiponectin. Circulation 1999; 100: $2473-$ 2476.

22. Ouchi N, Kihara S, Arita Y, Nishida M, Matsuyama A, Okamoto Y, et al. Adipocyte-derived plasma protein, adiponectin, suppresses lipid accumulation and class A scavenger receptor expression in human monocyte-derived macrophages. Circulation 2001; 103: 10571063.

23. Arita Y, Kihara S, Ouchi N, Maeda K, Kuriyama H, Okamoto Y, et al. Adipocyte-derived plasma protein adiponectin acts as a plateletderived growth factor-BB-binding protein and regulates growth 
factor-induced common postreceptor signal in vascular smooth muscle cell. Circulation 2002; 105: 2893-2898.

24. Kojima S, Funahashi T, Sakamoto T, Miyamoto S, Soejima H, Hokamaki J, et al. The variation of plasma concentrations of a novel, adipocyte derived protein, adiponectin, in patients with acute myocardial infarction Heart 2003; 89: 667.

25. Third Report of the National Cholesterol Education Program (NCEP) Expert Panel on Detection, Evaluation, and Treatment of High Blood Cholesterol in Adults (Adult Treatment Panel III) final report. Circulation 2002; 106: 3143-3421.

26. Norhammar A, Tenerz A, Nilsson G, Hamsten A, Efendic S, Ryden $\mathrm{L}$, et al. Glucose metabolism in patients with acute myocardial infarction and no previous diagnosis of diabetes mellitus: A prospective study. Lancet 2002; 359: 2140-2144.
27. Bartnik M, Malmberg K, Norhammar A, Tenerz A, Ohrvik J, Ryden L. Newly detected abnormal glucose tolerance: An important predictor of long-term outcome after myocardial infarction Eur Heart $J$ 2004; 25: 1990-1997.

28. The prevention or delay of type 2 diabetes. Diabetes Care 2002; 25: $742-749$.

29. Xiang AH, Peters RK, Kjos SL, Marroquin A, Goico J, Ochoa C, et al. Effect of pioglitazone on pancreatic beta-cell function and diabetes risk in Hispanic women with prior gestational diabetes. Diabetes 2006; 55: 517-522.

30. Maeda N, Takahashi M, Funahashi T, Kihara S, Nishizawa H, Kishida K, et al. PPARgamma ligands increase expression and plasma concentrations of adiponectin, an adipose-derived protein. Diabetes 2001; 50: 2094-2099. 